OPEN ACCESS

Edited by:

Wael Sabbah

Dental Institute, King's College

London, United Kingdom

Reviewed by:

Tamer M. S. Hifnawy,

Beni-Suef University, Egypt

Morenike Oluwatoyin Folayan,

Obafemi Awolowo University, Nigeria Haya Alayadi,

King's College London, United Kingdom

*Correspondence: Sharat C. Pani sharat@riyadh.edu.sa

Specialty section:

This article was submitted to

Children and Health

a section of the journal

Frontiers in Public Health

Received: 13 April 2019

Accepted: 01 July 2019

Published: 16 July 2019

Citation:

Al-Rafee MA, AlShammery AR AlRumikan AS and Pani SC (2019) A Comparison of Dental Caries in Urban and Rural Children of the Riyadh Region of Saudi Arabia.

Front. Public Health 7:195.

doi: 10.3389/fpubh.2019.00195

\section{A Comparison of Dental Caries in Urban and Rural Children of the Riyadh Region of Saudi Arabia}

\author{
Mohammed A. Al-Rafee ${ }^{1,2}$, Abdullah R. AlShammery ${ }^{1}$, Ali S. AlRumikan ${ }^{2}$ and \\ Sharat C. Pani ${ }^{\text {* }}$ \\ ${ }^{1}$ College of Dentistry, Riyadh Elm University, Riyadh, Saudi Arabia, ${ }^{2}$ Ministry of Health, Riyadh, Saudi Arabia
}

Background and Aim: The data presented in this study aimed to document caries prevalence and severity among a representative sample of 6,12 , and 15 years old Saudi Children.

Methodology: The study examined a total of 1,986 school children from 75 schools in urban and rural areas of Riyadh region, Saudi Arabia. Children having one or more dental caries lesion were used to determine prevalence of dental caries. Total number of decayed filled teeth (dft) was used to assess caries severity in the 6 years group while the DMFT was used to assess severity of dental caries at the 12 and 15 years age group.

Results: The caries prevalence was $85.77 \%$ in the 6 years group, $64.98 \%$ in the 12 years group, and $71.35 \%$ in the 15 years group. Mean dft was 1.4 (SD \pm 1.1$)$. The mean DMFT was $1.72(\mathrm{SD} \pm 0.49)$ at 12 years and 2.66 (SD \pm 0.55$)$ at 15 years. Both caries rate and severity were higher in rural areas than urban areas although the differences were not statistically significant.

Conclusion: The results of this study suggest that the prevalence and severity of dental caries in Saudi Arabia remain high, when compared to global averages.

Keywords: dental caries, dental public health, oral health, oral health surveys, Saudi Arabia (KSA)

\section{INTRODUCTION}

The countries of the Arabian Gulf have the highest per capital income in the Eastern Mediterranean region (EMRO) of the World Health Organization (WHO). Despite a high standard of living, the region has had a documented history of poor oral health with studies across the different countries attesting to high caries rates and poor oral hygiene (1).

Saudi Arabia is the largest country in this region and over the past decade there has been a great deal of interest in the evaluation of socioeconomic and cultural indicators of oral diseases and methods to quantify their impact $(2,3)$. While there have been attempts made to evaluate the oral health status in Saudi Arabia there has not been a unified calibrated examination of oral diseases in the Kingdom since 1993 (4). 
Saudi Arabia has a young population with persons under the age of 18 years accounting for nearly $40 \%$ of the population. The relationship between dental caries in childhood and caries experience as adults is a well-established one $(5,6)$. Numerous studies from across the region have confirmed the absence of proper oral hygiene and high caries rates in the samples of children studied (7-11). Furthermore, national statistics show that while over $80 \%$ of the Saudi population is concentrated in urban regions, the country is distributed over several rural areas spread across 2,250,000 sq. km; making a comparative assessment of urban and rural centers imperative (12).

The World Health Organization (WHO) recommends that oral health surveys in children be conducted at the ages of 5 years (to assess primary dentition), 12 years (to assess the permanent dentition soon after eruption), and 15 years (to assess the impact of dental caries on the erupted permanent dentition) (13). However, across the Saudi Arabia, children begin school at the age of 6 years making the recording of data in this age group more feasible.

The Oral Health Survey in children aged 6, 12, and 15 years was envisioned as a calibrated data collection exercise that would help clinicians, academics, and policy makers across the region better understand the nature of oral disease. The aim of this study was to determine caries prevalence and severity among children studying in the first grade ( $\sim 6$ years), 6th grade ( $\sim 12$ years), and 9 th grade $(\sim 15$ years) and compare the findings between children studying in urban and rural areas of Riyadh region, Saudi Arabia.

\section{METHODOLOGY}

\section{Ethical Approval}

Ethical approval for the study was obtained from the research center of the Riyadh Elm University (then Riyadh Colleges of Dentistry and Pharmacy) and permission to collect data was obtained from the Ministry of Health, Kingdom of Saudi Arabia and Ministry of Education, Kingdom of Saudi Arabia. Informed consent to examine the children was obtained from parents and assent obtained from children prior to examination.

\section{Allocation of Manpower and Calibration of Examiners}

As cultural norms of the Kingdom of Saudi Arabia do not permit the entry of males into female schools and vice-versa, two separate sets examiners ( 2 males and 2 females) were allocated for calibration. The examiners were calibrated for examination using the protocol laid down by the WHO (14). The inter-examiner kappa scores for primary teeth were 0.884 for decayed teeth (d), 0.891 for filled teeth (f), and 0.901 for the decayed and filled teeth $(\mathrm{dft})$. The kappa scores for permanent teeth were 0.921 for decayed teeth (D), 0.99 for missing teeth (M), and 0.912 for filled teeth with an overall score of 0.941 for the DMFT. The average intra-examiner scores for primary teeth were 0.945 for decayed, 0.881 for filled, and 0.916 for $\mathrm{dft}$.

\section{Sample Power Calculation and Distribution of the Sample}

The required sample based on an assumed true prevalence (based on existing literature) of 0.85 , assumed sensitivity and specificity of 0.8 and desired precision of 0.05 and confidence level of 0.95 was 1,843 children. The sample used existing census data from the Riyadh region to extrapolate the desired urban and rural sample (12). In order to account for variations in response rate a sample of 2,000 children 1,500 urban and 500 rural distributed over 81 schools (45 urban and 30 rural) were included in the survey using a stratified cluster sample model. In elementary schools junior grades and senior grades have separate head teachers even though they may often be included in the same physical space. For this reason, a school was defined as an administrative unit, rather than the physical school space. In rural areas there was a lower number of students per school, therefore the number of schools sampled was greater than the proportion of students sampled from each school. Children were identified by using attendance lists provided to the data coordinator (AaR) by the head teachers of each of the selected schools. The school clusters were stratified into three groups; first grade ( $\sim 6$ years), sixth grade $(\sim 12$ years $)$, and ninth grade ( $\sim 15$ years). An initial visit was followed up by a second visit to ensure that most of the targeted children could be included in the study. Of the targeted 2,000 children we were able to obtain data from 1,986 children giving us an actual power of 0.96 .

\section{Recording of Dental Caries}

WHO field examination protocol for examination of teeth was followed (14). Teeth were wiped with a dry piece of gauze before examination and a standardized portable LED with an intensity of. The number of decayed missing and filled permanent (DMF) and the decayed and filled primary (df) teeth were extrapolated from the raw data collected (13). Teeth with secondary caries (filled with decay) were considered as decayed teeth for the purpose of caries surveillance (13). No radiographs were used for the assessment of dental caries.

\section{Analysis of Dental Caries Data}

The prevalence of dental caries was estimated by the number of children with at least one active caries lesion as the numerator and the total number of children as the denominator. Children who had received at least one preventive and/or restorative dental visit were deemed to have had access to dental care. The mean $\mathrm{dft}$ and DMFT (for the 6 years group) and DMFT (for the 12 and 15 years age group) were compared between urban and rural children using the Mann Whitney $U$-Test. Caries prevalence was compared between the urban and rural populations using the Kolmogrov-Smirnov Z-test. Data was processed using the SPSS ver25 data processing software (IBM SPSS, IBM Corp., Armonk NY, USA).

\section{RESULTS}

\section{Demographic Data}

The sample comprised of 1,986 children (1,440 Urban, 466 Rural) of the 2,000 children whose parents were approached to 


\begin{tabular}{|c|c|c|c|c|c|}
\hline & & \multicolumn{4}{|c|}{ Percentage of population with one or more dental caries lesion } \\
\hline & & \multirow{2}{*}{\multicolumn{2}{|c|}{$\begin{array}{l}\text { Urban } \\
\text { Gender }\end{array}$}} & \multirow{2}{*}{\multicolumn{2}{|c|}{$\begin{array}{l}\text { Rural } \\
\text { Gender }\end{array}$}} \\
\hline & & & & & \\
\hline & & Male & Female & Male & Female \\
\hline \multirow[t]{3}{*}{ Age group } & 6 years -1 st grade & 87.21 & 84.64 & 86.93 & 85.21. \\
\hline & 12 years -6 th grade (permanent teeth only) & 62.51 & 66.85 & 63.21 & 68.22 \\
\hline & 15 years -9 th grade & 70.62 & 71.52 & 71.88 & 71.98 \\
\hline
\end{tabular}

participate in the study. The schools included 38 primary schools (first grade and sixth grade), 22 high schools (ninth grade), and 15 combined schools (where both junior and senior classes were in the same building). The sample comprised of 33 boys schools and 32 girls schools.

The age of the students in the 6 years age group ranged from 5.9 to 7.1 years (mean age $6.32, \mathrm{SD} \pm 0.37$ ) with no significant gender differences observed $(t=0.789, p=0.897)$. The age in the 12 years age group ranged from 11.9 to 13.4 years (mean age 12.45 years, $\mathrm{SD} \pm 0.41$ ) with no significant gender differences observed $(t=0.893, p=0.767)$. In 15 years age group the children were aged between 14.9 and 16.7 years (mean age 15.6 years, $\mathrm{SD} \pm 0.38$ ).

\section{Caries Prevalence}

Overall caries prevalence (percentage of the population with one or more dental caries lesion in a primary or permanent tooth) was $85.77 \%$ at 6 years, $64.98 \%$ at 12 years (for permanent teeth only), and $71.35 \%$ at 15 years (Table 1). There was no significant difference in caries prevalence between urban and rural areas at 6 years $(p=0.566), 12$ years $(p=0.432)$, or 15 years $(0.918)$. Similarly, no significant differences in caries prevalence were found between males and females at 6 years $(p=0.728), 12$ years $(p=0.654)$, or 15 years $(p=0.300)$.

\section{Dental Caries in Primary Teeth}

The decayed filled primary teeth (dft) was recorded only at the 6 years age group in keeping with WHO guidelines. The overall dft at 6 years was $1.4(\mathrm{SD} \pm 1.1)$. Females in rural areas had the lowest recorded $\mathrm{dft}(0.58)$. Despite the difference in meant $\mathrm{dft}$, there was a large standard deviation and the MannWhitney $U$-test found that the differences were not statistically significant (Table 2). When the urban and rural populations were compared it was observed that there was a lower dft observed in the urban (Mean 1.31, SD \pm 1.3 ) when compared to the rural (Mean 1.521, SD \pm 1.1 ). However, the Mann-Whitney $U$ Test showed that this difference was not statistically significant $(U=1577.00, t=0.752)$.

\section{Dental Caries in the Permanent Teeth}

Dental caries in the permanent teeth was measured using the DMFT index with mean DMFT of $1.72(\mathrm{SD} \pm 0.49)$ at 12 years and mean DMFT of 2.66 ( $\mathrm{SD} \pm 0.55)$. It was observed that females had lower DMFT scores at both 12 and 15 years. When gender differences were analyzed separately for the urban and
TABLE 2 | Comparison of mean dft between males and females in urban and rural areas.

\begin{tabular}{llcccc}
\hline Location & Gender & Mean & Std. deviation & Mann Whitney $\boldsymbol{~}$ & Sig $^{*}$ \\
\hline \multirow{2}{*}{ Urban } & Male & 1.58 & 1.16 & 1427.00 & 0.058 \\
& Female & 0.70 & 1.32 & & \\
\multirow{2}{*}{ Rural } & Total & 1.31 & 1.3 & & \\
& Male & 1.94 & 1.01 & 1672.00 & 0.132 \\
& Female & 0.43 & 0.58 & & \\
\hline
\end{tabular}

*Calculated using the Mann Whitney U-test.

rural population it was observed that rural females in the 12 years age group had a higher DMFT than their urban counterparts. However, gender differences were not statistically significant (Table 3). When urban and rural samples were compared it was observed that at both age groups the rural population had a greater DMFT score than the urban population, although these differences were not statistically significant (Table 4). There was a significant increase in the DMFT scores from 12 to 15 years. This was true for both the urban and rural population (Table 5).

\section{DISCUSSION}

Dental caries in Saudi Arabia is being recognized as one of the major public health problems facing the Kingdom (4). Despite the increase in publications on dental caries from Saudi Arabia, the last calibrated region wide study was conducted over two decades ago (15). The Riyadh region is one the largest regions of Saudi Arabia in terms of population. The study aimed to assess dental caries in the methodology prescribed by the WHO, as a precursor to a National Oral Health Survey.

Caries prevalence, or the number of children with at least one dental caries lesion is considered to be a reliable indicator of the extent of a dental caries epidemic. The caries prevalence rate seen in this study was lower than the previous nationwide study done in the 1990s which showed a prevalence of over $90 \%$. Overall the findings of this study show a slightly lower prevalence and severity level than a meta-analysis (4) of caries data but are in keeping with some newer studies in the $\operatorname{Kingdom}(14,16,17)$. While there has been a massive expansion in both preventive 
TABLE 3 | Comparison of DMFT between males and females at 12 and 15 years.

\begin{tabular}{lllccc}
\hline Location & Gender & Mean & Std. deviation & Mann Whitney $\boldsymbol{U}$ & Sig* $^{*}$ \\
\hline 12 years-urban & Male & 1.78 & 0.37 & 63.00 & 0.531 \\
& Female & 1.54 & 0.30 & & \\
& Total & 1.61 & 0.58 & & \\
12 years-rural & Male & 1.57 & 0.78 & 85.00 & 0.793 \\
& Female & 1.82 & 0.72 & & \\
& Total & 1.83 & 0.41 & & \\
12 years-overall & Male & 1.69 & 0.52 & 212.00 & 0.670 \\
& Female & 1.81 & 0.46 & & \\
& Total & 1.72 & 0.49 & & \\
15 years-urban & Male & 2.34 & 0.61 & 101.00 & 0.781 \\
& Female & 2.55 & 0.39 & & \\
& Total & 2.40 & 0.50 & & \\
15 years-rural & Male & 2.91 & 0.67 & 9.432 \\
& Female & 2.62 & 0.30 & & \\
& Total & 2.85 & 0.28 & & \\
15 years-overall & Male & 2.70 & 0.49 & 153.00 & 0.516 \\
& Female & 2.23 & 0.45 & & \\
& Total & 2.66 & 0.55 & & \\
\hline
\end{tabular}

"Calculated using the Mann Whitney U-test.

TABLE 4 | Comparison of DMFT between urban and rural areas at 12 and 15 years.

\begin{tabular}{llllll}
\hline Age group & \multicolumn{6}{c}{ Location } & Mean & Std. deviation & Mann Whitney $\boldsymbol{U}$ & $\mathbf{S i g}^{*}$ \\
\hline 12 years-6th grade & Urban & 1.61 & 0.58 & 484.00 & 0.412 \\
& Rural & 1.83 & 0.41 & & \\
Total & 1.72 & 0.49 & & \\
15 years-9th grade & Urban & 2.40 & 0.50 & 101.00 & 0.910 \\
& Rural & 2.85 & 0.28 & & \\
& Total & 2.66 & 0.55 & & \\
\hline
\end{tabular}

"Calculated using the Mann Whitney U-test.

Differences are not statistically significant.

and therapeutic dental services provided across Saudi Arabia over the past decade, the actual utilization of those services and whether they were responsible for the reduction in dental caries are beyond the scope of this study (18). The prevalence and severity of dental caries reported in the current study are in keeping with those of recent observational studies published from Saudi Arabia and $\operatorname{UAE}(5,17)$.

Despite this fact the rates are significantly higher than those reported from Europe and North America $(19,20)$. The rates reported in the study show that even though there appears to be some reduction in the caries prevalence and severity when compared to previous national surveys, the reduction is not as marked as has been shown in populations in Europe and North America (19-21). This fact should be a source of concern to public health planners in the country. Given the multifactorial nature of dental caries, there are could be several reasons for the low reduction in dental caries including poor oral hygiene, lack of parental awareness and inadequate preventive dental services;
TABLE 5 | Comparison of DMFT between the 12 and 15 years age groups.

\begin{tabular}{lccccc}
\hline Location & Gender & Mean & Std. deviation & Mann Whitney $\boldsymbol{U}$ & Sig $^{*}$ \\
\hline Urban & 12 years & 1.61 & 0.58 & 22.00 & $<0.001^{\text {** }}$ \\
& 15 years & 2.34 & 0.61 & & \\
Rural & 12 years & 1.83 & 0.41 & 17.00 & $<0.001^{\text {** }}$ \\
& 15 years & 2.85 & 0.28 & & \\
\multirow{2}{*}{ Overall } & 12 years & 1.72 & 0.49 & 64.00 & $<0.001^{\text {** }}$ \\
& 15 years & 2.66 & 0.55 & & \\
\hline
\end{tabular}

${ }^{*}$ Calculated using the Mann Whitney U-test.

${ }^{*}$ Differences were significant at $p<0.05$.

trends that have been documented by other studies from Saudi Arabia $(11,12,22)$.

One of the recommended protocols for the recording of dental caries in primary teeth is the recording of only decayed and filled primary teeth (dft), omitting the numbers of missing teeth in order to avoid the recording of teeth that might have been lost due to natural exfoliation. An alternative method is to record the decayed, extracted and filled teeth (deft). Our study population with primary teeth was aged between 6 and 7 years, an age when exfoliation of primary teeth begins, which was the reason for the use of $\mathrm{dft}$ over deft. One of the limitations of this approach, especially in a high caries population, is that teeth extracted due to dental caries are not recorded. Given this fact it is entirely possible that the dental caries prevalence in the primary teeth is actually higher than the currently reported levels. While this would be in keeping with some earlier studies done in Saudi Arabia $(2,3,16)$, it would go against more recent studies from Saudi Arabia that have shown rates of caries prevalence in primary teeth that are in keeping with the current study $(9,10)$.

The current study used population density (as documented by the Saudi National Census) as an indicator of Urban and Rural areas (15). Although there are several definitions available for Urban and Rural area, this was the measure used in the previous national study in Saudi Arabia and therefore chosen to enable accurate comparison to previous data (3). A significant difference from the previous data has been the reversal in urban and rural dental caries trends. Data from the 1990s had shown that rural Saudi children had significantly lower dental caries rates than their urban counterparts (22). While the current data showed no significant difference, it also showed that in many cases dental caries rates and severity in rural children was greater than their urban counterparts. One explanation for this shifting trend could be the fact that over the past two decades an easy availability of processed foods has meant a change in the eating patterns of rural Saudis (23).

One of the limitations of the study was that it only addressed the prevalence and severity of the disease without looking at sociodemographic variables such as parental education, economic status or family size. Furthermore, the current paper only reports the dental caries status and does not examine the oral hygiene practice or other individual reasons for the high dental caries. 


\section{CONCLUSION}

The results of the study suggest that dental caries prevalence and severity in Saudi Arabia remain high when compared to global averages. Further analysis of the data, especially sociodemographic variables is needed to determine effective caries planning strategies for the Kingdom.

\section{DATA AVAILABILITY}

Additional data will be made available upon reasonable request to the authors.

\section{ETHICS STATEMENT}

This study was carried out in accordance with the recommendations of the Institutional Review Board of the Riyadh Elm University, with written informed consent from the parents of all subjects. The parents of all subjects gave written informed consent in accordance with the Declaration of Helsinki. The protocol was approved by the Institutional Review Board of the Riyadh Elm University.

\section{REFERENCES}

1. Al-Ismaily $\mathrm{M}, \mathrm{Al}$-Busaidy $\mathrm{K}$, Al-Khussaiby $\mathrm{A}$. The progression of dental disease in Omani schoolchildren. Int Dent J. (2004) 54:409-10. doi: 10.1111/j.1875-595X.2004.tb0 0019.x

2. al Shammery A, el Backly M, Guile EE. Permanent tooth loss among adults and children in Saudi Arabia. Commun Dent Health. (1998) 15:277-80.

3. al-Shammery AR. Caries experience of urban and rural children in Saudi Arabia. $J$ Public Health Dent. (1999) 59:60-4. doi: 10.1111/j.1752-7325.1999.tb0 3236.x

4. Wyne AH. Caries prevalence, severity, and pattern in preschool children. $J$ Contemp Dent Pract. (2008) 9:24-31.

5. Al-Bluwi GS. Epidemiology of dental caries in children in the United Arab Emirates. Int Dent J. (2014) 64:219-28. doi: 10.1111/idj. 12114

6. Hashim R, Williams SM, Thomson WM, Awad MA. Caries prevalence and intra-oral pattern among young children in Ajman. Commun Dent Health. (2010) 27:109-13.

7. Jordan R, Bodechtel C, Hertrampf $\mathrm{K}$, Hoffmann $\mathrm{T}$, Kocher $\mathrm{T}$, Nitschke I, et al. The Fifth German Oral Health Study (Funfte Deutsche Mundgesundheitsstudie, DMS V) - rationale, design, and methods. BMC Oral Health. (2014) 14:161. doi: 10.1186/1472-683114-161

8. Petersen P. Improvement of global oral health-the leadership role of the World Health Organization. Commun Dent Health. (2010) 27:194-8.

9. Khan SQ, Khan NB, Arrejaie AS. Dental caries. A meta-analysis on a Saudi population. Saudi Med J. (2013) 34:744-9.

10. Al Agili DE. A systematic review of population-based dental caries studies among children in Saudi Arabia. Saudi Dent J. (2013) 25:3-11. doi: 10.1016/j.sdentj.2012.10.002

11. Togoo RA, Al-Rafee MA, Kandyala R, Luqam M, Al-Bulowey MA. Dentists' opinion and knowledge about preventive dental care in saudi arabia: a

\section{AUTHOR CONTRIBUTIONS}

MA-R was responsible for the conduct of the study, supervision of examiners, and overall logistical support. ARA was responsible of the conceptualization of the study and calibration of the examiners. ASA was responsible for co-ordination between the schools and the logistical requirements for the study. SP was responsible for the analysis and interpretation of the data. All authors contributed to the preparation of the manuscript.

\section{FUNDING}

This study was supported by logistical support from the Riyadh Elm University. No external funds were used for the study.

\section{ACKNOWLEDGMENTS}

The authors would like to thank the Research Center of the Riyadh Elm University for statistical and logistical support for the calibration of examiners. We would like to thank the Ministry of Health, Kingdom of Saudi Arabia and Ministry of Education, Kingdom of Saudi Arabia for facilitating the examination of the children. We are grateful to Dr. Nawaf Al Habib, Dr. Yahya Al Qahtani, Dr. Loloh Al Selemi, and Dr. Rania Fadan for their contribution as calibrated examiners for the study.

nationwide cross-sectional study. J Contemp Dent Pract. (2012) 13:261-5. doi: 10.5005/jp-journals-10024-1134

12. Wyne A. Oral hygiene practices and first dental visit among early childhood caries children in Riyadh. J Pakistan Dent Assoc. (2003) 15:161-66

13. Wyne A, al-Dlaigan $\mathrm{Y}$, Khan N. Caries prevalence, oral hygiene and orthodontic status of Saudi Bedouin children. Indian J Dent Res. (2001) 12:194-8.

14. World Health Organization. Oral Health Surveys: Basic Methods. World Health Organization (2013).

15. CDSI Annual Statistics Information. (2012). Available online at: http://www. cdsi.gov.sa/english/

16. al-Shammery AR, Guile EE, el-Backly M. Prevalence of caries in primary school children in Saudi Arabia. Commun Dent Oral Epidemiol. (1990) 18:320-1. doi: 10.1111/j.1600-0528.1990.tb 00089.x

17. Alhabdan YA, Albeshr AG, Yenugadhati N. Prevalence of dental caries and associated factors among primary school children: a population-based cross-sectional study in Riyadh, Saudi Arabia. Environ Health Prev Med. (2018) 23:60. doi: 10.1186/s12199-0180750-z

18. AlHumaid J, El Tantawi M, AlAgl A, Kayal S, Al Suwaiyan Z, Al-Ansari A, et al. Dental visit patterns and oral health outcomes in Saudi Children. Saudi J Med Med Sci. (2018) 6:89-94.doi: 10.4103/sjmms.sjmms_103_17

19. Dye BA, Mitnik GL, Iafolla TJ, Vargas CM. Trends in dental caries in children and adolescents according to poverty status in the United States from 1999 through 2004 and from 2011 through 2014. $J$ Am Dent Assoc. (2017) 148:550-65.e7. doi: 10.1016/j.adaj.2017. 04.013

20. Davies GM, Neville J, Jones K, White S. Why are caries levels reducing in five-year-olds in England? Br Dent J. (2017) 223:515-19. doi: 10.1038/sj.bdj.2017.836

21. Santamaria RM, Schmoeckel J, Basner R, Schuler E, Splieth CH. Caries Trends in the Primary Dentition of 6- to 7-Year-old Schoolchildren in Germany from 1994 to 2016: Results from the German National 
Oral Health Surveys in Children. Caries Res. (2019). doi: 10.1159/0005 00854. [Epub ahead of print].

22. Aldossary MS, Alamri AA, Alshiha SA, Hattan MA, Alfraih YK, Alwayli HM. Prevalence of dental caries and fissure sealants in the first permanent molars among male children in Riyadh, Kingdom of Saudi Arabia. Int J Clin Pediatr Dent. (2018) 11:365-70. doi: 10.5005/jp-journals-100 05-1541

23. Moradi-Lakeh M, El Bcheraoui C, Afshin A, Daoud F, AlMazroa MA, Al Saeedi M, et al. Diet in Saudi Arabia: findings from a nationally representative survey. Public Health Nutr. (2017) 20:1075-81. doi: 10.1017/S1368980016 003141
Conflict of Interest Statement: The authors declare that the research was conducted in the absence of any commercial or financial relationships that could be construed as a potential conflict of interest.

Copyright (c) 2019 Al-Rafee, AlShammery, AlRumikan and Pani. This is an openaccess article distributed under the terms of the Creative Commons Attribution License (CC BY). The use, distribution or reproduction in other forums is permitted, provided the original author(s) and the copyright owner(s) are credited and that the original publication in this journal is cited, in accordance with accepted academic practice. No use, distribution or reproduction is permitted which does not comply with these terms. 